\title{
Estrułura e Funções de um Centro de Programação e Informática
}

JOÃO JORGE DA CUNHA

Professor do Instituto de Estudos Avançados

\section{INTRODUÇÃO}

O modêlo de organização aqui apresentado permite a implantação de uma Central de Informática, abrangendo não sòmente os aspectos de estocagem, seleção e transmissão de informações, mas, também, o treinamento de recursos humanos destinados à pesquisa em ciência da informação e aos centros de informática.

Em sua elaboração preferiu-se mencionar e descrever as funções que devem compor todo o órgão em vez de, rigidamente, estruturar as unidades que o compõem. Assim, cada setor poderá ter a estrutura mais adequada às condições locais de sua área de ação, tornando-se o modêlo proposto aplicável a um maior número de casos específicos.

Entre as diversas funções do CEPI deve haver a mais ampla comunicabilidade para que as metas e planos setoriais se integrem num só plano, harmônico e equilibrado, evitando-se uma indesejável justaposição dos mesmos.

\section{PROFISSIONAIS DO CEPI}

A experiência tem demonstrado que os grupos de trabalho dêsses órgãos são de caráter interprofissional. Deve ser considerada a necessidade organizacional nos aspectos social, econômico, físico e institucional. Para isso são necessários conhecimentos heterogêneos que profissionais da mesma formação disciplinar não poderiam ter. A metodologia que estamos apreSentando fornece uma indicação precisa das necessidades do órgão em têrmos de pessoal técnico especializado. 
O número e qualificação dos técnicos necessários serão equacionados em função das atividades do CEPI.

Recomenda-se, porém, que, ao se cogitar da organização do Centro, preveja-se a inclusão inicial de, pelo menos, um técnico para cobrir cada uma das seguintes áreas: economia, educação, administração, urbanismo, saúde, ciências básicas, humanidades e, principalmente, informática.

\section{PROGRAMAÇÃO}

O equipamento do CEPI deve ter enorme capacidade de processamento, com projeções de teleprocessamento; deve ser capaz de adaptar suas programações aos requisitos de cada segmento de sistema de informação estruturado; deve ter grande capacidade de ampliação para atender aos processos de crescimento das necessidades no campo da informática.

\section{INFORMÁTICA}

Considerando-se que a informática é um conjunto de conhecimentos que podem ser úteis e mesmo indispensáveis em qualquer tomada de decisão, o modêlo proposto deverá:

a) possibilitar a utilização de um banco central de dados e de recuperação de informações com as capacidades de atualização de registros e de computação;

b) permitir o uso ou a recuperação das informações pela introdução de linguagem que se adapte às necessidades específicas;

c) fornecer a chave para aproveitamento das potencialidades latentes contidas nos dispositivos de armazenamento de dados, potencialidades que permitirão aos sistemas usuários projetar seu futuro através da simulação, da pesquisa e da análise.

Elementos especializados prepararão os relatórios, gráficos etc., que servirão de apoio às decisões dos usuários.

Caberá, também, ao CEPI a função de organizar um serviço especializado de microfilmagem para atender às suas necessidades e às dos usuários de seus serviços. Todos os levantamentos e estudos realizados deverão ser microfilmados e ordenados, mediante programações. Dêsse modo, ter-se-á, a partir da organização do serviço, informações precisas sôbre qualquer setor de atuação do CEPI em poucos segundos. 
Os encarregados dêsse serviço deverão, ainda, através do Serviço de Relações Públicas do CEPI manter contatos com instituições dedicadas à informática, nacionais e estrangeiras, a fim de obter publicações especializadas, planos, notícias e informações que permitam uma constante atualização de conhecimentos por parte de seus técnicos.

\section{ADAPTAÇÃO}

O modêlo proposto é flexível e modular, adaptável às necessidades e operações correntes nas emprêsas e nos órgãos públicos e capaz de crescer acompanhando o crescimento dos sistemas usuários. Pode descrever tôdas as gradações, desde um simples sistema de recuperação de informações a uma rêde de computação servindo virtualmente a cada aspecto do complexo do usuário.

\section{COMO FUNCIONA}

As vantagens oferecidas pelo CEPI são, naturalmente, proporcionais à carga de trabalho que Ihe fôr atribuída.

Se o CEPI serve sòmente a um setor (mão-de-obra, por exemplo), os programas a serem desenvolvidos consistirão, fundamentalmente, de registros de performances. Esses registros permitem a elaboração de relatórios de atividades, de faIhas no processo de trabalho, o confronto de dados de admissão, impressão de transcrições diversas, além de uma grande variedade de outras informações do setor.

\section{PESQUISA}

A pesquisa é um instrumento importante no desenvolvimento de qualquer sistema social. A pesquisa no campo da informática será sugerida, naturalmente, pelos vários departamentos do CEPI. Torna-se, assim, possivel o desenvolvimento cuidadoso de programas envolvendo os próprios recursos humanos gerados pelo CEPI.

\section{ANÁLISE PELA SIMULAÇÃO}

A simulação consiste no desenvolvimento de um modêlo descritivo de um sistema organizacional que se queira desenvolver, envolvendo testes de avaliação de planos alternativos e estratégias que permitam decisões antecipadas.

É necessário destacar, entretanto, que, através da simulação, a incidência de erros e acertos para a seleção final realiza- 
se, na sua totalidade, no computador, não tendo, assim, tradução na realidade, a não ser que se chegue a uma decisão e, a partir daí, se passe à execução do plano escolhido.

\section{AUTONOMIA}

Como resultado de sua modularidade, o CEPI possibilitará a todos os seus departamentos manter sua autonomia através de fichários individualizados em que se baseiam os projetos.

Como resultado de planejamento, fica-se livre de qualquer necessidade de programação que indicie, pesquise, recupere ou até mesmo comunique-se com os fichários ou dispositivos de entrada/saída que não atenda a um grande número de casos. Recuperam-se dados através do uso de programas de alto nível, semelhantes quanto à linguagem, e pode-se, também, determinar as características do resultado desejado em casos especificos.

\section{RECURSOS HUMANOS}

Aspecto do modêlo de bastante interêsse será o da geração de recursos humanos de alto nível através de cursos de diferentes níveis.

O CEPI poderá manter, em caráter permanente, cursos de diferentes níveis, visando ao treinamento e à formação de gerentes, pesquisadores e consultores em informática.

A implantação dêsses cursos deve seguir às seguintes recomendações:

a) é indispensável ter uma equipe de professôres exclusivamente dedicada aos cursos;

b) deve-se evitar dispersão de esforços resultante da organização de cursos sem o pessoal capacitado para ministrá-los;

c) cada curso deve ser objeto de planejamento meticuloso, na base de projeto, do qual conste, obrigatòriamente:
1) categoria;
2) objetivos;
3) número de vagas;
4) requisitos para inscrição e matrícula;
5) normas para seleção dos candidatos inscritos;
6) início e término;
7) número de períodos; 
8) duração dos períodos;

9) carga horária do curso;

10) disciplinas obrigatórias, optativas e disciplinas de pré-requisitos;

11) carga horária de cada disciplina ou unidade de ensino, que deverá ser múltiplo do valor horário da unidade de crédito;

12) sumário de cada disciplina;

13) créditos de cada disciplina;

14) metodologia a ser empregada;

15) processo de avaliação;

16) instrumento da avaliação;

17) documento que será fornecido aos alunos que concluírem o curso.

\section{NECESSIDADES DE RECURSOS HUMANOS}

A grande expansão empresarial que se vem verificando no Grande Rio e no Grande São Paulo, nos últimos anos, requer um número crescente de profissionais criadores, capazes de desenvolver novas técnicas, processos, métodos e equipamentos no campo fascinante da informática.

Por outro lado, com a criação do Plano Nacional de Informações e a resolução de implantar os serviços absolutamente necessários das Divisões de Segurança e Informações em cada Ministério, foram ampliadas extraordinàriamente as necessidades de recursos humanos de vários níveis para atuarem no campo da informática.

Os programas objeto dêstes comentários têm o objetivo de dar aos nossos graduados, principalmente, a oportunidade de estenderem os seus conhecimentos num setor vital para o desenvolvimento do País - o da ciência da informação.

Ao lado de uma formação geral, estão projetadas as especializações que atenderão às necessidades do desenvolvimento do País e aos interêsses da administração pública nos seus diferentes níveis.

\section{MERCADO DE TRABALHO}

Quando um indivíduo se propõe a realizar estudos especializados em certa área, é natural que procure saber as oportunidades de trabalho que se the oferecem. Dependendo de seu 
talento e de alguns ajustamentos ditados pelos programas, as oportunidades para o pessoal treinado em ciência da informação se apresentam, tanto no Govêrno como nas emprêsas privadas, em quatro áreas:

1) treinamento de mão-de-obra para os sistemas de informação;

2) pesquisa sôbre ciência da informação;

3) administração de sistemas de informação;

4) consultoria em ciência da informação e produção, análise e transmissão de informação.

Seria razoável admitir uma necessidade anual de 100 especialistas em cada uma dessas áreas.

\section{ESTRUTURA DOS CURSOS}

O Regimento dos Cursos foi organizado de forma a abranger cursos de Programação, Análise de Sistemas e Informática. Segundo êsse regimento, a concessão do grau de especialista requer:

a) um mínimo de créditos, incluindo os créditos de aulas e laboratório e os créditos dos trabalhos individuais;

b) média global obtida nos programas não inferior a 3 , ou seja, B;

c) demonstração de conhecimentos em pelo menos um idioma estrangeiro, à escolha da Coordenação-Geral dos Cursos;

d) demonstração de conhecimentos nas áreas definidas no programa aprovado pela Coordenação-Geral do CEPI;

e) apresentação de trabalho individual.

A critério da Coordenação-Geral dos Cursos podem ser aceitos até 5 (cinco) créditos de outras instituições.

Nos cursos de pós-graduação são classificados os que apresentam as seguintes qualificações:

a) ter díploma de curso superior numa das áreas indicadas pela Coordenação-Geral dos Cursos;

b) ter demonstrado aptidão para estudos pós-graduação na área da informática, através de testes de seleção;

c) demonstrar conhecimentos suficientes de Português e de uma língua estrangeira, pelo menos. 


\section{SUGESTOEES DE PROGRAMAS}

a) Curso de pós-graduação em informática: 100 - Curso Básico 100.1 - Metodologia da Ciência.

100.2 - Estatística.

100.3 - Matemática.

100.4 - Econometria.

100.5 - Contabilidade.

100.6 - Pesquisa operacional.

200 - Tecnologia.

200.1 - Programação em computadores eletrônicos.

200.2 - Estrutura dos grandes fichários.

200.3 - Análise de sistemas.

200.4 - Rêde de computadores.

200.5 - Máquinas seqüenciais e automata.

200.6 - Sistemas de informações.

300 - Heurística.

300.1 - Teoria dos jogos.

300.2 - Cognição.

300.3 - Lógica e mapas cognitivos.

300.4 - Cibernética e Sistemas Adaptativos.

300.5 - Sistemas de comunicação e contrôle.

300.6 - O processo heurístico.

400 - Administração de Sistemas de Informação.

400.1 - Teoria da organização.

400.2 - Organização de sistemas de informação.

400.3 - Gerência de sistemas de informação.

400.4 - Administração de sistemas de informação.

400.5 - Utilização de Serviços de Informação.

500 - Treinamento profissional.

500.1 - Didática da comunicação.

500.2 - Consultoria.

500.3 - Gerência da informação.

500.4 - Pesquisa.

600 - Temas para trabalhos individuais.

600.1 - Conhecimentos de Matemática relacionados com álgebra moderna, matrizes, cálculo numérico, estatística, teoria da informação e pesquisa operacional. 
600.2 - Teoria da informação, relacionada, essencialmente, com subsistemas de aquisição, análise, armazenamento, busca, separação e impressão de informações e/ou codificação, estocagem e organização de arquivo, o processo dos jogos ou recuperação, distribuição, avaliação e processos de realimentação.

600.3 - Sistemas de computação e aplicação de computadores na administração e em problemas de transferência de informações.

600.4 - Sistemas de rêde, centros de informações especializados e o tipo de sistema que êsses centros operam; estratégias de busca e fornecimento de informações.

b) Curso de graduação: Análise de Sistemas.

100 - Introdução.

100.1 - Os analistas.

100.2 - Caracteres dos analistas.

100.3 - Tipos de analistas.

100.4 - Terminologia.

100.5 - Problemas e limitações.

200 - As fases da análise.

200.1 - Definição.

200.2 - Coleta de dados.

200.3 - Análise do sistema atual.

200.4 - Projeto do nôvo sistema.

200.5 - Documentação.

200.6 - Implantação.

200.7 - Operação.

300 - Coleta de dados.

300.1 - Plano de trabalho.

300.2 - Técnicas de levantamento.

300.3 - Níveis de levantamento.

300.4 - Elementos a coletar.

300.5 - Registro dos fatos.

300.6 - Roteiro do levantamento.

400 - Análise.

400.1 - Como analisar.

400.2 - Como usar os formulários de análise. 
400.3 - Resultados da análise.

400.4 - Sistemograma ou fluxograma de procedimentos.

400.5 - Técnicas especiais para construção do sistemograma.

400.6 - Fluxograma de processamento ou processograma.

400.7 - Estudo de viabilidade.

400.8 - Roteiro de análise.

500 - Projeto do nôvo sistema.

500.1 - Definição da filosofia do projeto.

500.2 - Desenvolvimento de soluções para a atividade principal.

500.3 - Tarefas.

500.4 - Elementos.

500.5 - Contrôles. 500.51 - Sôbre os dados de entrada. 500.52 - Internos. 500.53 - De saída.

500.6 - Limitações.

500.7 - Desenvolvimento de soluções para as atividades secundárias.

500.8 - Integração.

500.9 - Estudo das alternativas.

500.10 - Avaliação dos sistemas.

500.11 - Arquivos e registros.

600 - Implantação.

600:1 - Planejamento.

600.2 - Preparação.

600.3 - Registro de progresso.

600.4 - Conversão.

600.5 - Finalização.

600.6 - Avaliação dos resultados obtidos.

600.7 - Comunicação analista-programador.

600.8 - Criação do Centro de Informática.

700 - Sistemas serviços.

700.1 - As aplicações.

700.2 - A organização necessária para funcionamento ótimo do sistema de informações.

700.3 - Funcionamento do sistema. 
c) Cursos de treinamento, aperfeiçoamento etc.

100 - Programação científica.

100.1 - Fundamentos de sistema de computador.

100.2 - Introdução à programação.

100.3 - FORTRAN

200 - Programação comercial.

200.1 - Fundamentos de sistema de computador.

200.2 - Introdução à programação.

200.3 - RPG

200.4 - Assembler

200.5 - COBOL

300 - Outros cursos de programação.

15. CONSULTORIA

Sem afetar seus trabalhos rotineiros, a versatilidade do CEPI oferece condições para o planejamento de sistemas informativos das entidades usuárias.

Todos os projetos e programas que forem submetidos à apreciação do CEPI serão examinados com imparcialidade e acompanhados rigorosamente segundo normas contratuais.

O CEPI fornecerá informações aos órgãos públicos e às emprêsas, podendo manter e ampliar seus serviços com receitas provenientes de prestação de serviços. O Sistema ERIC, por exemplo, no Office of Education dos Estados Unidos da América, tem uma receita anual de 10.000 .000 de dólares com êsses serviços.

Em suas funções de consultoria o CEPI teria uma função importantíssima no campo da informática: a de seleção da informação, a fim de atender à crescente especialização e ao aproveitamento do tempo disponível dos especialistas.

Aspecto significativo da consultoria em informática será 0 da estimativa de custos de implantação dos sistemas de informação.

"The problem of choosing the best information storage and retrieval system, diz Keith, * involves a consideration of the

* Keith Jr., M.R. - A General Evaluation Model for an Information Storage and Retrieval System, Journal of the American Society for Information Science, Vol. 21, no 4, 1970. 
operational characteristics of information storage and retrieval systems relative to information requirements. Using these considerations, evaluations can be based upon decision theoretical considerations. The lack of such a decision theoretical basis for the study of the operation of these systems makes it difficult to evolve a well defined approach even to the gathering of evidence upon which to base a choice of a particular system."

\section{SISTEMAS DE CUSTOS}

Consideremos, por exemplo, um sistema de custos para um modêlo geral de "storage and retrieval information".

100 - Custo total do Sistema de informação (CT) constituído de:

100.1 - Custo de iniciação (ci);

100.2 - Custo de manutenção $(\mathrm{cm})$;

100.3 - Custo de alimentação (ca);

100.4 - Custo de operação do computador (co);

100.5 - Custo de saída da informação (cs);

100.6 - Custos tributários e promocionais (ctp)

O custo total será expresso pela seguinte equação:

$$
\mathrm{CT}=\mathrm{ci}+\mathrm{cm}+\mathrm{ca}+\mathrm{co}+\mathrm{cs}+\mathrm{ctp}
$$

Consideremos os custos parciais começando pelos custos de iniciação. Êsses custos envolvem os custos de implementação (imp), os de pessoal técnico e administrativo (st, de staff), os da equipe (treinamento) para usar o nôvo equipamento (eq) a ser alugado ou comprado e a conversão do sistema de informação existente para o adotado (con). Teremos, assim, para o cálculo dos custos de iniciação, a equação seguinte:

$$
c i=c_{i m p}+c_{s t}+c_{\text {eq }}+c_{\text {con }}
$$

Os custos de manutenção $(\mathrm{cm})$ podem ser desdobrados nos seguintes fatôres: custos de atualização dos fichários (at) e custos de impressão das informações (impr). Ambos êsses custos dependem naturalmente da quantidade de informações 
que se deseja estocar e da que se deseja usar. A equação dêsses fatôres é a seguinte:

$$
\mathrm{cm}=\mathrm{c}_{\text {at }}+\mathrm{c}_{\text {impr }}
$$

Os custos de alimentação do sistema (ca) compreendem os custos de codificação (co) e os custos de processamento (pro). A maior ou menor complexidade dos questionários determinará as variações de custos dêsse parâmetro. A equação dos custos de alimentação é a seguinte:

$$
\mathrm{ca}=\mathrm{c}_{\mathrm{co}}+\mathrm{c}_{\text {pro }}
$$

O fator operação do sistema pode envolver custos que variam com o tempo exigido para o processamento dos questionários (opr) e urgência (ur) no atendimento do usuário. Quando êste último pode esperar pela resposta, o custo por unidade de tempo de operação do computador diminui consideràvelmente pela possibilidade de utilização de intervalos de programação ou de tempo ocioso do computador. A equação para os custos de operação é a seguinte:

$$
\mathrm{co}=\mathrm{c}_{\mathrm{opr}}+\mathrm{c}_{\mathrm{ur}}
$$

Os custos de saída de informação são determinados pela complexidade dos títulos e número de páginas (tp), bem como pelo tempo de listagem das informações e fornecimento das informações aos solicitantes (list). Podemos caracterizar êsses custos na seguinte equação:

$$
\mathrm{cs}=\mathrm{c}_{\mathrm{tp}}+\mathrm{c}_{\text {list }}
$$

Outros custos devem ser acrescentados além dos que foram relacionados - são os custos tributários e promocionais (ctp). Correspondem ao somatório dos impostos (imp), taxas (tx), obrigações (obr), royalties ( $r t)$ e de serviços promocionais e publicitários ( $\mathrm{pp}$ ) que incidem sôbre o sistema. A equação é:

$$
\mathrm{ctp}=\mathrm{c}_{\mathrm{imp}}+\mathrm{c}_{\mathrm{tx}}+\mathrm{c}_{\mathrm{obr}}+\mathrm{c}_{\mathrm{rt}}+\mathrm{c}_{\mathrm{pp}}
$$


17. BIBLIOGRAFIA CONSULTADA

ASIS - Journal of the American Society for Information Science, vol. 21, no 4 - 1970: vários artigos, principalmente "Education in Information Science", por Jack Belzer, pág. 269.

ASHBY, W. R. - Uma Introdução à Cibernética, Ed. Perspectiva, S. P., 1970.

CABLE, E. J. et al - The Physical Sciences, Prentice Hall, New Jersey, 5th ed., 1969.

KENT, Allen - Textbook on Mechanized Information Retrieval, John Wiley, New York, 2nd ed., 1966.

MEADOW, Charles T. - The Analysis of Information Systems, John Wiley, New York, 1967.

SCIENTIFIC AMERICAN BOOK - Information, W. H. Freeman, São Francisco, 1968.

TAUBE, Mortiner - Computers and Common Sense, Columbia University Press, New York, 1961. 